\title{
Model analysis of the world data on the pion transition form factor
}

\author{
S. Noguera ${ }^{\mathrm{a}}$ and V. Vento ${ }^{\mathrm{b}}$ \\ Departamento de Fisica Teorica and Instituto de Física Corpuscular, Universidad de Valencia-CSIC, E-46100 Burjassot Valencia, \\ Spain
}

Received: 5 July 2012 / Revised: 11 September 2012

Published online: 23 October 2012

(C) The Author(s) 2012. This article is published with open access at Springerlink.com Communicated by S. Hands

\begin{abstract}
We discuss the impact of recent Belle data on our description of the pion transition form factor based on the assumption that a perturbative formalism and a nonperturbative one can be matched in a physically acceptable manner at a certain hadronic scale $Q_{0}$. We discuss the implications of the different parameters of the model in comparing with world data and conclude that within experimental errors our description remains valid. Thus we can assert that the low $Q^{2}$ nonperturbative description together with an additional $1 / Q^{2}$ term at the matching scale have a strong influence on the $Q^{2}$ behavior up to very high values of $Q^{2}$.
\end{abstract}

New data of the pion transition form factor $(\pi \mathrm{TFF})$ from the Belle Collaboration have just appeared [1]. These data, above $10 \mathrm{GeV}^{2}$, are smaller in magnitude than the previous BABAR data [2], which generated considerable excitement. The question to unveil is the scale of asymptotia. BABAR data, taken at face value, implied that asymptotic QCD behavior lies at much higher $Q^{2}$ than initially expected [3,4]. Belle data seem to lower that scale. We show here that our scheme can accomodate easily all data without changing the physical input.

At the time of the BABAR data we developed a formalism to calculate the $\pi \mathrm{TFF}$ [5], which consists of three ingredients: i) a low energy description of the $\pi \mathrm{TFF}$; ii) a high energy description of the $\pi \mathrm{TFF}$; iii) a matching condition between the two descriptions at a scale $Q_{0}$ characterizing the separation between the two regimes. For the low energy description we took a parametrization of the low energy data to avoid model dependence at $Q_{0}$. The high energy description of the $\pi \mathrm{TFF}$, defined by the pion Distribution Amplitude ( $\pi \mathrm{DA})$, contains Quantum Chromodynamic (QCD) evolution from $Q_{0}$ to any higher $Q$, a mass cut-off to make the formalism finite, and an additional $1 / Q^{2}$ term which leads to modifications of the matching condition.

Let us recall some aspects of the formalism. The high energy description, to lowest order in perturbative QCD, for the transition form factor in the process $\pi^{0} \rightarrow \gamma \gamma^{*}$ in terms of the pion distribution amplitude $(\pi \mathrm{DA})$, is given

\footnotetext{
a e-mail: Santiago.Noguera@uv.es

b e-mail: Vicente.Vento@uv.es
}

by

$$
Q^{2} F\left(Q^{2}\right)=\frac{\sqrt{2} f_{\pi}}{3} \int_{0}^{1} \frac{\mathrm{d} x}{x+\frac{M^{2}}{Q^{2}}} \phi_{\pi}\left(x, Q^{2}\right) .
$$

We follow the proposal of Polyakov [6] and Radyushkin [7] and introduce a cut-off mass $M$ to make the expression finite. $Q^{2}=-q^{2}, q_{\mu}$ is the momentum of the virtual photon, $\phi_{\pi}\left(x, Q^{2}\right)$ is $\pi \mathrm{DA}$ at the $Q^{2}$ scale and $f_{\pi}=0.131 \mathrm{GeV}$. In this expression, the $Q^{2}$ dependence appears through the QCD evolution of the $\pi \mathrm{DA}$.

Despite the fact that several models reproduce the low energy data, in order to have a model-independent expression for the form factor at low virtualities, we adopted a monopole parametrization of the $\pi \mathrm{TFF}$ in the low energy region as

$$
F^{L E}\left(Q^{2}\right)=\frac{F(0)}{1+a \frac{Q^{2}}{m_{\pi^{0}}^{2}}} .
$$

with $F(0)=0.273(10) \mathrm{GeV}^{-1}$ and $a=0.032$ (4) [8], determined from the experimental study of $\pi^{0} \rightarrow \gamma e^{+} e^{-}[9]$.

Additional power corrections can be introduced in eq. (1) by adding to the lowest-order calculation a term proportional to $Q^{-2}$,

$$
Q^{2} F\left(Q^{2}\right)=\frac{\sqrt{2} f_{\pi}}{3} \int_{0}^{1} \frac{\mathrm{d} x}{x+\frac{M^{2}}{Q^{2}}} \phi_{\pi}\left(x, Q^{2}\right)+\frac{C_{3}}{Q^{2}} .
$$



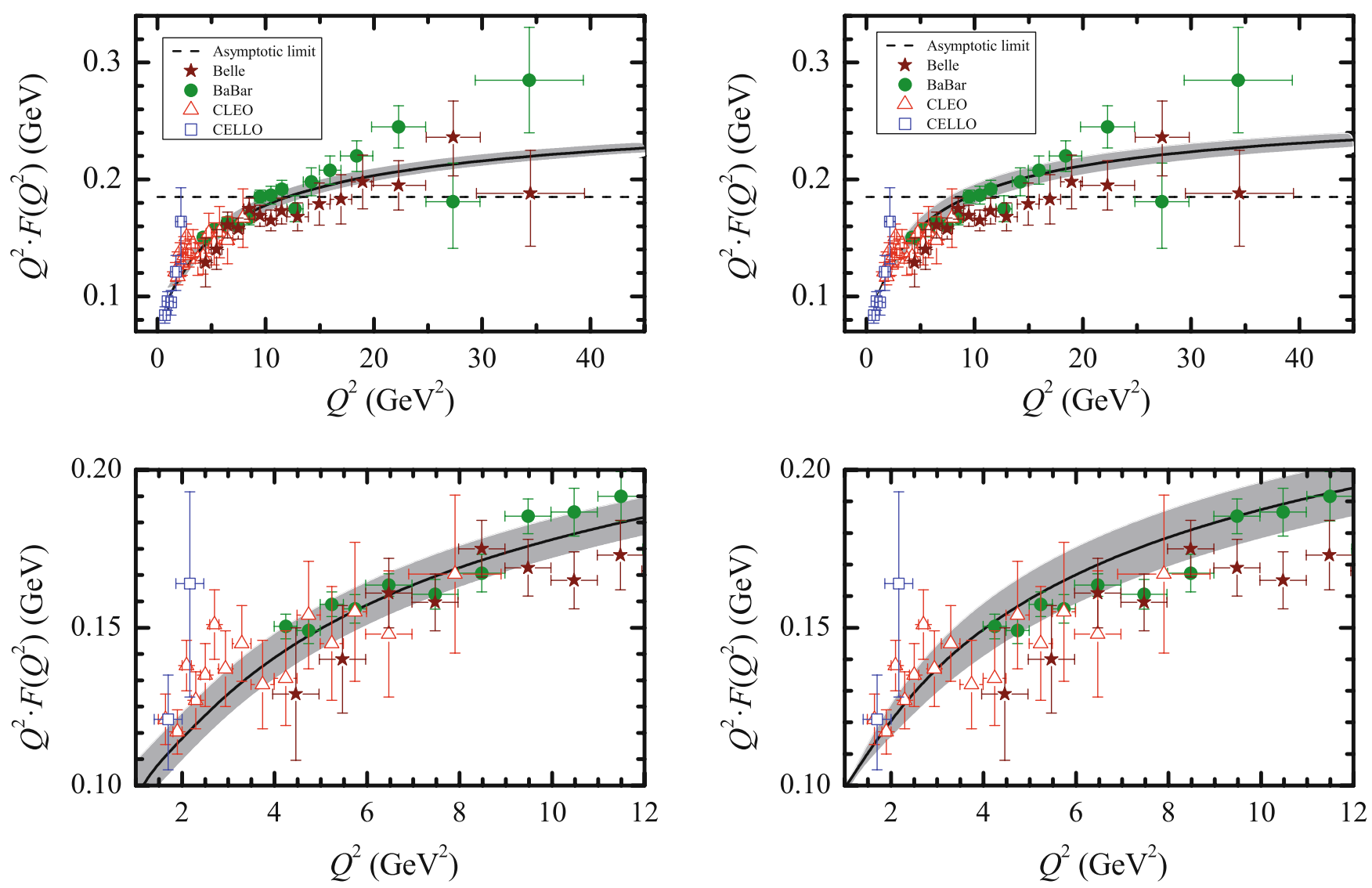

Fig. 1. We show the result for the transition form factor in our formalism for $M=0.690 \mathrm{GeV}, a=0.032$ and $C_{3}=$ $2.9810^{-2} \mathrm{GeV}^{3}$ and take the matching point at $Q_{0}=1 \mathrm{GeV}$ (solid line). The band region results from the indeterminacy in $\Delta a= \pm 0.004$. The lower plot shows the detailed behavior for low virtuality. Data are taken from CELLO [10], CLEO [11], BABAR [2] and Belle [1].

Using a constant $\pi$ DA the matching condition becomes [5],

$$
\frac{\sqrt{2} f_{\pi}}{3} \ln \frac{Q_{0}^{2}+M^{2}}{M^{2}}+\frac{C_{3}}{Q_{0}^{2}}=\frac{F(0) Q_{0}^{2}}{1+a \frac{Q_{0}^{2}}{m_{\pi^{0}}^{2}}},
$$

with $Q_{0}=1 \mathrm{GeV}$. This equation allows to determine $M$, once we have fixed the value of $C_{3}$.

We analyze here the sensitivity of the data to the various parameters involved. We keep as close as possible to our previous fit analyzing the data with respect to small variations in the low virtuality parameter $a$ and in the higher-twist parameter $C_{3}$. In fig. 1 we show the effect of the precision in the determination of the monopole parametrization. We see that as $a$ increases from 0.032 to 0.036 , i.e. within the error bars, the $\pi \mathrm{TFF}$ decreases. The sensitivity to $C_{3}$ is shown in fig. 2 and we note that as the value of $C_{3}$ increases from $C_{3}=0.9910^{-2} \mathrm{GeV}^{3}$, which corresponds to a $10 \%$ contribution to the form factor at $Q_{0}$, to $2.9810^{-2} \mathrm{GeV}^{3}$, which corresponds to a $30 \%$ contribution, again the value of the $\pi \mathrm{TFF}$ decreases. Thus a small increase in $a$ and $C_{3}$ moves our result to-

Fig. 2. We show the result for the transition form factor in our formalism for $M=0.620 \mathrm{GeV}, a=0.032$ and the value of $C_{3}=1.9810^{-2} \mathrm{GeV}^{3}$ corresponding to $20 \%$ of the contribution at the matching point at $Q_{0}=1 \mathrm{GeV}$ (solid line). The band region gives the variation of the results due in $\pm 10 \%$ in the contribution of higher twist. The lower plot shows the detailed behavior for low virtuality. Data are taken from CELLO [10], CLEO [11], BABAR [2] and Belle [1].

ward the Belle data. Finally, in fig. 3, we plot the better fit $\left(\chi^{2} /\right.$ dof $\left.=1.21\right)$ taking into account all the world data which corresponds to $a=0.032$ with the $C_{3}$ term at the $30 \%$ value. We stress that there is no strong correlation between $a$ and $C_{3}$ as long as $a$ is kept within its experimental error bars. Thus the fit is quite stable with respect to the parameters of the low energy model.

The fit to the data is excellent with a very small variation of the $1 / Q^{2}$ contribution at $Q_{0}$ from previous fit, i.e. from $20 \%$ to $30 \%$. It must be said, before entering the discussion of this fit, that in our previous work [5] we pointed out that the average value of the highest energy data points of BABAR were too large, a conclusion reached also by other analyses [12-14]. In fig. 3 we show not only the fit for $30 \%$ contribution of $C_{3} / Q^{2}$ at $Q_{0}$, but its behavior for higher values of $Q^{2}$. As can be seen, also stressed in our previous work, this contribution is small in size. However, and this an important outcome of our analysis, it is instrumental in fixing the initial slope at the matching point, which determines, after evolution, the high energy behavior of the form factor. 

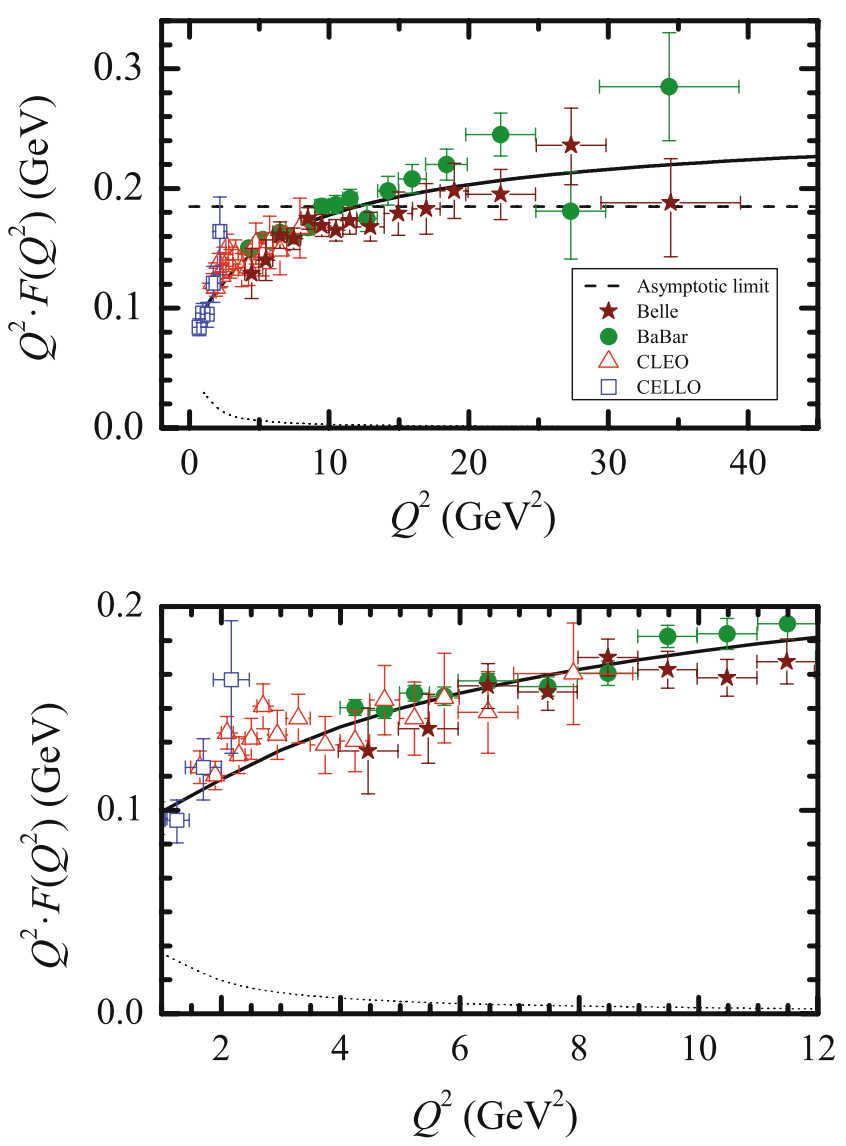

Fig. 3. We show the result for the transition form factor in our formalism for $M=0.690 \mathrm{GeV}, a=0.032$ and the value of $C_{3}=2.9810^{-2} \mathrm{GeV}^{3}$ corresponding to $30 \%$ of the contribution at the matching point at $Q_{0}=1 \mathrm{GeV}$ (solid line). The lower plot shows the detailed behavior for low virtuality. The dotted curve represents the higher-twist contribution. Data are taken from CELLO [10], CLEO [11], BABAR [2] and Belle [1].

In our opinion the Belle data confirm the BABAR result that the $\pi \mathrm{TFF}$ crosses the asymptotic QCD limit. This limit is well founded under QCD assumptions, but nothing is known of how this limit is reached, if from above or from below. BABAR and Belle data suggest that the limit is exceeded around $10-15 \mathrm{GeV}$. Our calculation is consistent with this result. The necessary growth of the $\pi \mathrm{TFF}$ between $5-10 \mathrm{GeV}$ to achieve this crossing is in our case an indication of nonperturbative behavior and $C_{3} / Q^{2}$ contribution at low virtuality. The determination of the crossing point is a challenge for any theoretical model and therefore, the precise experimental determination of it is of relevance. Many models fail to achieve this crossing because their pion DA is defined close to its asymptotic form.

The pion DA can be expressed as a series in the Gegenbauer polynomials,

$$
\begin{aligned}
\phi_{\pi}\left(x, Q^{2}\right)= & 6 x(1-x) \\
\times & \left(1+\sum_{n(\text { even })=2}^{\infty} a_{n}\left(Q^{2}\right) C_{n}^{3 / 2}(2 x-1)\right) .
\end{aligned}
$$

We can compare different models by looking at the values of the coefficients of the expansion $a_{n}\left(Q^{2}\right)$. In our case, at $Q^{2}=1 \mathrm{GeV}^{2}$ many $a_{n}$ coefficients are significant, but we focus our attention on a few terms: $a_{2}=0.389$, $a_{4}=0.244$ and $a_{6}=0.179$. At $Q^{2}=4 \mathrm{GeV}^{2}$ we obtain the values $a_{2}=0.307, a_{4}=0.173$ and $a_{6}=0.118$, which are close to those obtained by Polyakov [6]. Consistently, our result for the $\pi \mathrm{TFF}$ is similar to that obtained in ref. [6]. At $Q^{2}=5.76 \mathrm{GeV}^{2}$ we obtain $a_{2}=0.292$, $a_{4}=0.161$ and $a_{6}=0.108$, which are very different from those of ref. [15]. These author use for their fit BABAR data for the $\eta \mathrm{TFF}[16]$, together with the pion data. It is therefore not a surprise that these authors come to a different conclusion, namely, that the Belle and the BABAR data cannot be reproduced to the same level of accuracy within the Light Cone Sum Rules approach [17]. However, in an extension of the ideas developed in the present paper to the $\eta$ case studied in ref. [18] looking at the state $|q\rangle=\frac{1}{2}(|u \bar{u}\rangle+|d \bar{d}\rangle)$ a very different structure of the $a_{n}$ coefficients to that of the pion arises. At $Q^{2}=1 \mathrm{GeV}^{2}$, the values of the coefficients are $a_{2}=0.134$ and $a_{4}=0.352$ or, equivalently, at $Q^{2}=5.76 \mathrm{GeV}^{2}$ we have $a_{2}=0.101$ and $a_{4}=0.232$. Therefore, that study does not supports the combined use of both datasets.

We have developed a formalism to describe the $\pi \mathrm{TFF}$ on all experimentally accessible range, and hopefully beyond. The formalism is based on a two energy scale description. The formulation in the low energy scale is nonperturbative, while that of the high energy scale is based on perturbative QCD. The two descriptions are matched at an energy scale $Q_{0}$ called hadronic scale $[19,20]$. We stress the crucial role played by the nonperturbative input at the level of the low energy description. It is an important outcome of this calculation the role played by the $1 / Q^{2}$ power correction term in determining the slope of the data at high $Q^{2}$, despite the fact that they do almost not contribute to the value of the $\pi \mathrm{TFF}$.

We have used a flat $\pi \mathrm{DA}$, i.e. a constant value for all $x[7,6]$, which with our normalization becomes $\phi(x)=1$. Our choice has been motivated by chiral symmetry [5]. Model calculations, Nambu-Jona-Lasinio (NJL) [21-24] and the "spectral" quark model [25], give a constant $\pi \mathrm{DA}$. The $\pi$ TFF calculated in these models, however, overshoots the data [26], emphasizing the importance of QCD evolution.

The calculation shown proves that the BABAR and Belle results can be accommodated in our scheme, which only uses standard QCD ingredients and low energy data. Moreover, at the light of our results, we confirm that at $40 \mathrm{GeV}^{2}$ we have not yet reached the asymptotic regime which will happen at higher energies.

We would like to thank A.V. Pimikov and M.V. Polyakov for useful comments. This work has been partially funded by the Ministerio de Economía y Competitividad and EU FEDER under contract FPA2010-21750-C02-01, by Consolider Ingenio 2010 CPAN (CSD2007-00042), by Generalitat Valenciana: Prometeo/2009/129, by the European Integrated Infrastructure Initiative HadronPhysics3 (Grant number 283286). 
Open Access This is an open access article distributed under the terms of the Creative Commons Attribution License (http://creativecommons.org/licenses/by/3.0), which permits unrestricted use, distribution, and reproduction in any medium, provided the original work is properly cited.

\section{References}

1. The Belle Collaboration (S. Uehara et al.), arXiv:1205.3249 [hep-ex].

2. The BABAR Collaboration (B. Aubert et al.), Phys. Rev. D 80, 052002 (2009) arXiv:0905.4778 [hep-ex].

3. G.P. Lepage, S.J. Brodsky, Phys. Rev. D 22, 2157 (1980).

4. V.L. Chernyak, A.R. Zhitnitsky, Phys. Rep. 112, 173 (1984).

5. S. Noguera, V. Vento, Eur. Phys. J. A 46, 197 (2010) arXiv:1001.3075 [hep-ph].

6. M.V. Polyakov, JETP Lett. 90, $228 \quad$ (2009) arXiv:0906.0538 [hep-ph].

7. A.V. Radyushkin, Phys. Rev. D 80, 094009 (2009) arXiv:0906.0323 [hep-ph].

8. Particle Data Group Collaboration (K. Nakamura et al.), J. Phys. G 37, 075021 (2010).

9. Particle Data Group (C. Amsler et al.), Phys. Lett. B 667, 1 (2008).

10. CELLO Collaboration (H.J. Behrend et al.), Z. Phys. C 49, 401 (1991).

11. CLEO Collaboration (J. Gronberg et al.), Phys. Rev. D 57, 33 (1998) arXiv:hep-ex/9707031.
12. S.V. Mikhailov, N.G. Stefanis, Mod. Phys. Lett. A 24, 2858 (2009) arXiv:0910.3498 [hep-ph].

13. A.E. Dorokhov, Phys. Part. Nucl. Lett. 7, 229 (2010) arXiv:0905.4577 [hep-ph].

14. A.E. Dorokhov, Nucl. Phys. Proc. Suppl. 198, 190 (2010) arXiv:0909.5111 [hep-ph].

15. A.P. Bakulev, S.V. Mikhailov, A.V. Pimikov, N.G. Stefanis, Phys. Rev. D 86, 031501 (2012) arXiv:1205.3770 [hep$\mathrm{ph}$.

16. BABAR Collaboration (P. del Amo Sanchez et al.), Phys. Rev. D 84, 052001 (2011) arXiv:1101.1142 [hep-ex].

17. A.P. Bakulev, S.V. Mikhailov, A.V. Pimikov, N.G. Stefanis, Phys. Rev. D 84, 034014 (2011) arXiv:1105.2753 [hep$\mathrm{ph}$.

18. S. Noguera, S. Scopetta, Phys. Rev. D 85, 054004 (2012) arXiv:1110.6402 [hep-ph].

19. M. Traini, A. Mair, A. Zambarda, V. Vento, Nucl. Phys. A 614, 472 (1997).

20. S. Noguera, V. Vento, Eur. Phys. J. A 28, 227 (2006) arXiv:hep-ph/0505102.

21. I.V. Anikin, A.E. Dorokhov, L. Tomio, Phys. Lett. B 475, 361 (2000) hep-ph/9909368.

22. M. Praszalowicz, A. Rostworowski, Phys. Rev. D 64, 074003 (2001) arXiv:hep-ph/0105188.

23. E. Ruiz Arriola, W. Broniowski, Phys. Rev. D 66, 094016 (2002) arXiv:hep-ph/0207266.

24. A. Courtoy, S. Noguera, Phys. Rev. D 76, 094026 (2007) arXiv:0707.3366 [hep-ph].

25. E. Ruiz Arriola, W. Broniowski, Phys. Rev. D 67, 074021 (2003) arXiv:hep-ph/0301202.

26. W. Broniowski, E.R. Arriola, arXiv:0910.0869 [hep-ph]. 